\title{
POSTER
}

\section{Ostéoradionécrose et odontologie : mise au point}

\section{Belhaj K, El Harti K, El Wady W}

Service de chirurgie orale, faculté de médecine dentaire, Rabat MAROC

Les cancers des voies aéro-digestives supérieures représentent, de par leur fréquence (10\% de l'ensemble des cancers) et leur taux de mortalité (de l'ordre de $70 \%$ à 5 ans), un enjeu de santé publique majeur.

La radiothérapie constitue l'un des traitements fondamentaux de nombreux cancers. À côté de son objectif qui est de bloquer l'évolution tumorale et de réduire la masse tumorale, l'irradiation du tissu osseux et des tissus de recouvrement constitue le facteur initial du développement d'une ostéoradionécrose (ORN), la complication la plus sérieuse survenant après traitement par irradiation.

Le traitement de cette pathologie est long et complexe. II fait appel à la coopération de toute l'équipe médicale : radiothérapeute, chirurgien maxillo-facial, médecin dentiste et médecin ORL.

Ce travail constitue une mise au point sur l'actualité concernant l'ostéoradionécrose et la prise en charge odontologique des patients avant, pendant et après la radiothérapie. 